\title{
Extension of the generalized multipole technique to three-dimensional anisotropic scatterers
}

\author{
Nicolas B. Piller and Olivier J. F. Martin \\ Laboratory of Field Theory and Microwave Electronics, Swiss Federal Institute of Technology, ETH-Zentrum, 8092 Zurich, Switzerland
}

Received January 5, 1998

\begin{abstract}
New expansions are derived for the simulation of three-dimensional anisotropic scatterers with the generalized multipole technique (GMT). This extension of the GMT makes possible the investigation of subtle phenomena such as the interaction of light with realistic crystals or magneto-optic materials. (c) 1998 Optical Society of America

OCIS codes: $\quad 290.0290,160.1190,230.3810$.
\end{abstract}

The generalized multipole technique (GMT) is a powerful frequency-domain technique for the simulation of scattering problems. ${ }^{1-4}$ It is used for the study of a broad range of applications, for example, near-field microscopy ${ }^{5}$ scattering by particles, ${ }^{6}$ and design of antennas. $^{7}$ The aim of this Letter is to extend this technique to handling three-dimensional anisotropic scatterers in a way similar to that by which we recently handled the two-dimensional case. ${ }^{8}$

The basic idea of the GMT is to decompose the scatterer into homogeneous domains and to represent the field in each domain by an expansion fulfilling Maxwell's equations inside the corresponding domain. The boundaries between the different domains are discretized with $D$ points, and the six boundary conditions for the electric and the magnetic fields at each discretization point are enforced, which leads to a system of equations with $N$ unknowns and six $D$ equations, where $N$ is the total number of expansion parameters. The number of discretization points $D$ is usually chosen such that the system of equations becomes strongly overdetermined. In this way, the residual error on the boundary conditions at the discretization points gives a good measure of the overall result accuracy. A more detailed description of the GMT is available in the literature. ${ }^{9,10}$ The expansion that is generally used for any simply connected closed domain is the Bessel multipole expansion (BME). For isotropic media, this expansion is defined by use of the scalar Bessel multipole, ${ }^{11}$

$$
\psi_{l}^{m}(\mathbf{r})=j_{l}(k|\mathbf{r}|) Y_{l}^{m}(\theta, \phi),
$$

where $j_{l}(k|\mathbf{r}|)$ is the spherical Bessel function, $Y_{l}^{m}(\theta, \phi)$ is the spherical harmonics, and $k$ is the wave number in the medium. The electric fields of the electric and the magnetic Bessel multipoles of degree $l$ and order $m$, respectively, are

$$
\begin{aligned}
{ }^{e} \mathbf{E}_{l}^{m}(\mathbf{r}) & =\nabla \times \nabla \times\left[\mathbf{r} \psi_{l}^{m}(\mathbf{r})\right], \\
{ }^{h} \mathbf{E}_{l}^{m}(\mathbf{r}) & =i k \nabla \times\left[\mathbf{r} \psi_{l}^{m}(\mathbf{r})\right] .
\end{aligned}
$$

The BME of order $L$ is then the linear combination of the Bessel multipoles given in Eqs. (2) and (3) with $l=1 \ldots L$ and $m=-l \ldots l$.

0146-9592/98/080579-03\$15.00/0
To obtain the Bessel multipole for an anisotropic medium, we first express the Bessel multipole for an isotropic medium with a plane-wave expansion. In other words, we search the plane-wave distribution representing the multipole. Then, the same distribution is used, but with the plane waves corresponding to the anisotropic medium. To the best of our knowledge, a plane-wave expansion of the Bessel multipole has never been presented in the literature. Nevertheless, its derivation is similar to that of the plane-wave expansion for the Hankel multipole, which was brilliantly described by Bobbert and Vlieger. ${ }^{12}$

The starting point of their derivation [Ref. 12, Eq. (6.1)] was the spectral representation of the scalar Hankel multipole [Eq. (1), with the Hankel function instead of the Bessel function]. We therefore represent the scalar spherical solution with the Bessel radial function [Eq. (1)] in a similar manner ${ }^{13}$ :

$$
\psi_{l}^{m}(\mathbf{r})=\frac{i^{-1}}{4 \pi} \int_{S^{\prime}} \exp \left(i k \hat{k}^{\prime} \mathbf{r}\right) Y_{l}^{m}\left(\theta^{\prime}, \phi^{\prime}\right) \mathrm{d} \Omega^{\prime},
$$

where $\hat{k}^{\prime}$ is a unit vector pointing in the $\left(\theta^{\prime}, \phi^{\prime}\right)$ direction, the integral domain $S^{\prime}$ represents the entire domain of the spherical variables $\theta^{\prime}$ and $\phi^{\prime}\left(\theta^{\prime}=\right.$ $\left.0 \ldots \pi, \phi^{\prime}=0 \ldots 2 \pi\right)$, and $\mathrm{d} \Omega^{\prime}$ is the corresponding solid angle $\left[\mathrm{d} \Omega^{\prime}=\sin \left(\theta^{\prime}\right) \mathrm{d} \phi^{\prime} \mathrm{d} \theta^{\prime}\right]$. Using a derivation similar to that of Bobbert and Vlieger, based on Debye potentials and Hertz vectors, we find that the planewave representation of the Bessel multipoles becomes

$$
\begin{aligned}
{ }^{e} \mathbf{E}_{l}^{m}(\mathbf{r})= & \frac{k}{4 \pi} \int_{S^{\prime}} \exp \left(i m \phi^{\prime}\right)\left[\tilde{V}_{l}^{m} \mathbf{e}_{\theta^{\prime}, \phi^{\prime}}(\mathbf{r})\right. \\
& \left.+i \tilde{U}_{l}^{m} \mathbf{e}_{\theta^{\prime}, \phi^{\prime}}^{\boldsymbol{\phi}^{\prime}}(\mathbf{r})\right] \mathrm{d} \Omega^{\prime}, \\
{ }^{h} \mathbf{E}_{l}^{m}(\mathbf{r})= & \frac{k}{4 \pi} \int_{S^{\prime}} \exp \left(i m \phi^{\prime}\right)\left[i \tilde{U}_{l}^{m} \mathbf{e}_{\theta^{\prime}, \phi^{\prime}}^{\boldsymbol{\theta}^{\prime}}(\mathbf{r})\right. \\
& \left.-\tilde{V}_{l}^{m} \mathbf{e}_{\theta^{\prime}, \phi^{\prime}}^{\boldsymbol{\phi}^{\prime}}(\mathbf{r})\right] \mathrm{d} \Omega^{\prime},
\end{aligned}
$$

where $\tilde{U}_{l}^{m}$ and $\tilde{V}_{l}^{m}$ are sums of Legendre functions that depend on $\theta^{\prime}$ and are defined in Eq. (8.5) of Ref. 12 and $\mathbf{e}_{\theta, \phi}^{\boldsymbol{\theta}}(\mathbf{r})$ and $\mathbf{e}_{\theta, \phi}^{\phi}(\mathbf{r})$ are the electric fields of the plane waves propagating in the $(\theta, \phi)$ direction and polarized in the $\boldsymbol{\theta}$ and the $\boldsymbol{\phi}$ directions, respectively.

(C) 1998 Optical Society of America 
As we want to use this plane-wave representation for anisotropic media, we need the plane-wave solution for an anisotropic medium. Such a plane wave is a solution of an eigenvalue problem where the eigenvectors represent the polarization and the eigenvalues represent the corresponding propagation constants. ${ }^{14}$ For each $(\theta, \phi)$ direction, two plane waves, $\mathbf{e}_{\theta, \phi}^{\mathrm{pw}_{1}}(\mathbf{r})$ and $\mathbf{e}_{\theta, \phi}^{\mathrm{pw} w_{2}}(\mathbf{r})$, are then obtained, but their polarizations usually do not coincide with the $\boldsymbol{\theta}$ and $\boldsymbol{\phi}$ polarizations of the plane waves in Eqs. (5) and (6). We therefore define pseudo $\boldsymbol{\alpha}$-polarized plane waves, where $\boldsymbol{\alpha}$ stands for $\boldsymbol{\theta}$ or $\boldsymbol{\phi}$, as a linear combination of $\mathbf{e}_{\theta, \phi}^{\mathrm{pw}_{1}}(\mathbf{r})$ and $\mathbf{e}_{\theta, \phi}^{\mathrm{pw}_{2}}(\mathbf{r})$ :

$$
\mathbf{e}_{\theta, \phi}^{\alpha}(\mathbf{r})=a_{\theta, \phi}^{\alpha} \mathbf{e}_{\theta, \phi}^{\mathrm{pw}_{1}}(\mathbf{r})+b_{\theta, \phi}^{\alpha} \mathbf{e}_{\theta, \phi}^{\mathrm{pw}_{2}}(\mathbf{r}),
$$

where the coefficients $a_{\theta, \phi}^{\alpha}$ and $b_{\theta, \phi}^{\alpha}$ are set such that the corresponding displacement field is $\boldsymbol{\alpha}$ polarized at the multipole origin $(\mathbf{r}=0)$. We call the field in Eq. (7) a pseudo plane wave because the sum cannot be written as a plane wave, as the propagation constants of $\mathbf{e}_{\theta, \phi}^{\mathrm{pw}_{1}}(\mathbf{r})$ and $\mathbf{e}_{\theta, \phi}^{\mathrm{pw}_{2}}(\mathbf{r})$ are different.

Finally, we obtain the Bessel multipoles for anisotropic media by replacing the isotropic plane waves in Eqs. (5) and (6) with the pseudo $\boldsymbol{\theta}$ - and $\boldsymbol{\phi}$ polarized plane waves for anisotropic media [Eq. (7)]. Unfortunately, the integrals in Eqs. (5) and (6) then cannot be evaluated analytically anymore but must be performed numerically by approximation with a sum. It is important to note that no additional error is introduced by this numerical integration. Indeed, each plane wave fulfills Maxwell's equations, and so does the multipole. From a numerical view point, we notice that if we first discretize the spherical integration domain into colatitudes and then discretize each colatitude linearly the summation over each colatitude represents a discrete Fourier transform and therefore its speed can be increased with a fast Fourier transform.

Another way to represent the field inside the anisotropic domain is to expand it directly into plane waves. This expansion, which we call the plane wave expansion (PWE), is a linear combination of plane-wave couples $\left[e_{\theta, \phi}^{\mathrm{pw}_{1}}(\mathbf{r}), e_{\theta, \phi}^{\mathrm{pw}_{2}}(\mathbf{r})\right]$ propagating in all directions. Its main advantage is that the evaluation of the field is much less time consuming than with the $\mathrm{BME}$, since no summation or fast Fourier transform is required.

The convergence of the BME and the PWE was studied for spherical scatterers by use of solutions evaluated with the coupled-dipole approximation ${ }^{15,16}$ as a reference. The same behavior as for the twodimensional case was observed, ${ }^{8}$ i.e., a rapid convergence as soon as the number of expansion parameters was large enough to represent the field. In each case, fewer parameters are required for the BME than for the PWE to achieve a given accuracy, which implies a smaller system of equations for the BME.

As a first numerical example, we consider the scattering of a magneto-optic $\mathrm{Dy}_{7} \mathrm{Co}_{5}$ sphere of radius $250 \mathrm{~nm}$ illuminated by a plane wave propagating in the $\hat{z}$ direction. The scatterer is magnetized by a static magnetic field pointing in the $\hat{z}$ direction, and the wavelength of the incident field is $633 \mathrm{~nm}$. For this wavelength, the permittivity of $\mathrm{Dy}_{7} \mathrm{Co}_{5}$ is ${ }^{17}$

$$
\overleftrightarrow{\epsilon}=\left[\begin{array}{ccc}
\epsilon_{d} & -\zeta \epsilon_{\text {off }} & 0 \\
\zeta \epsilon_{\text {off }} & \epsilon_{d} & 0 \\
0 & 0 & \epsilon_{d}
\end{array}\right]
$$

where $\epsilon_{d}=3.01+7.35 i, \epsilon_{\text {off }}=0.056-0.0078 i$, and $\zeta$ is a parameter representing the magnetization. This parameter vanishes for no magnetization and is equal to 1 at saturation. The parameters $S_{3}$ and $S_{4}$ of the scattering amplitude matrix ${ }^{18}$ are represented in Fig. 1 for the scattering directions $(\theta=-\pi \ldots \pi, \phi=0)$ as a function of $\zeta . \quad S_{3}$ and $S_{4}$ represent the coupling of the $s$-polarized incident field with the $p$-polarized scattered field and the $p$-polarized incident field with the $s$-polarized scattered field, respectively. When no magnetization is applied $(\zeta=0), S_{3}$ and $S_{4}$ vanish, and no coupling is observed. When magnetization is applied, coupling appears, and $S_{3}$ and $S_{4}$ increase with increasing magnetization (Fig. 1). This coupling
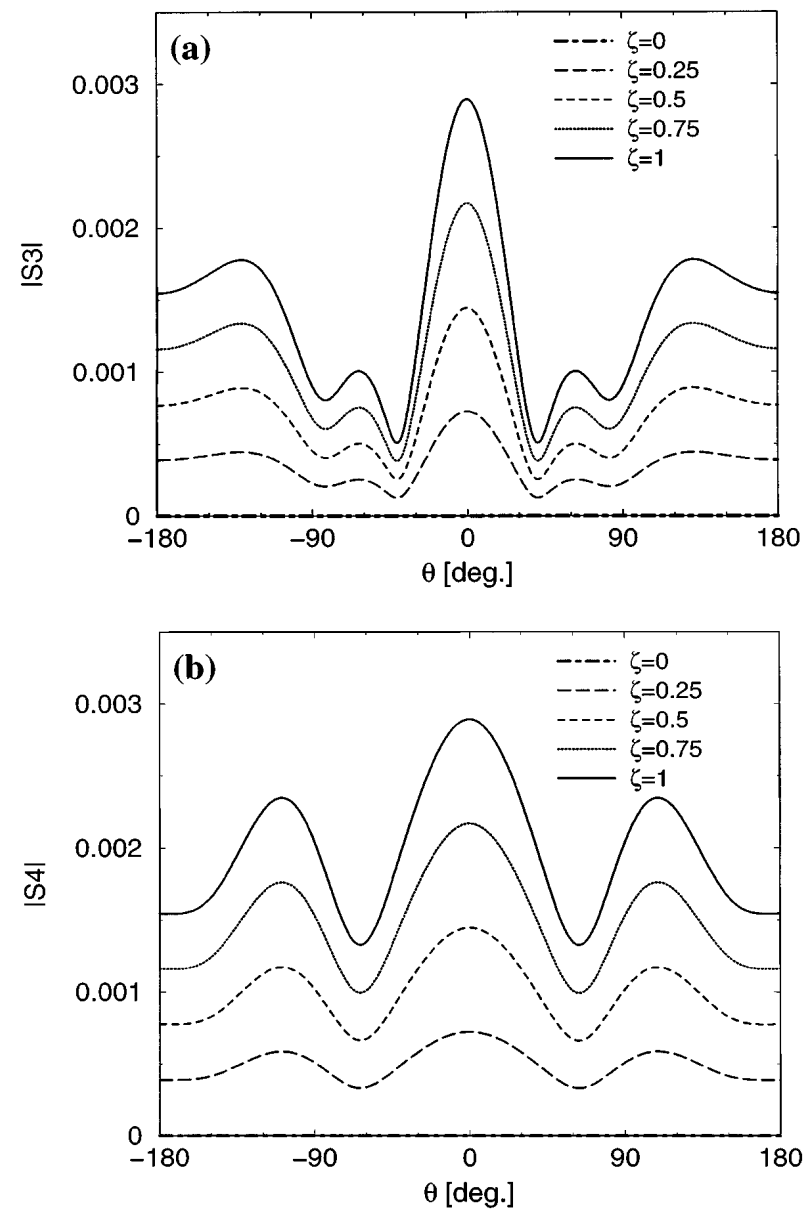

Fig. 1. Parameters (a) $S_{3}$ and (b) $S_{4}$ of the scattering amplitude matrix for a magneto-optic $\mathrm{Dy}_{7} \mathrm{Co}_{5}$ sphere of radius $250 \mathrm{~nm}$. The incident field is a plane wave of wavelength $633 \mathrm{~nm}$ propagating in the $\hat{z}$ direction. The scatterer is magnetized in the $\hat{z}$ direction. The parameters $S_{3}$ and $S_{4}$ are represented for the scattering directions $(\theta=$ $-\pi \ldots \pi, \phi=0$ ) for different magnetization parameters $\zeta$ between zero magnetization $(\zeta=0)$ and saturation $[\zeta=1$; Eq. (8)]. 


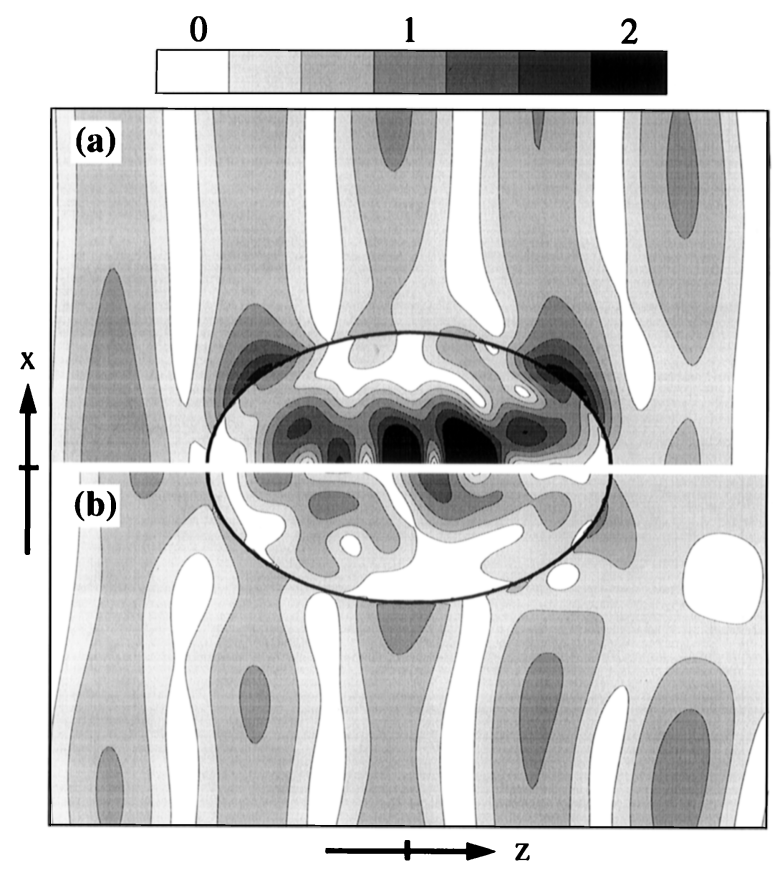

Fig. 2. Amplitude of the electric field at time $t=0$ for a $\mathrm{TiO}_{2}$ ellipsoid. The major and minor axes of the ellipsoid are 900 and $600 \mathrm{~nm}$, respectively. The scatterer is illuminated by a unit plane wave of wavelength $633 \mathrm{~nm}$, polarized in the $\hat{x}$ direction and propagating in the $\hat{z}$ direction. The crystal's ordinary (extraordinary) permittivity is 6.67 (8.24). The crystal's principal axis is parallel to either (a) the $\hat{x}$ axis or (b) the $\hat{y}$ axis.

represents a rotation of the polarization plane and is known as the Kerr effect. This effect was studied for the one-dimensional case. ${ }^{19,20}$ Precise knowledge of the angular dependence of this effect for a finitesize sample, as illustrated in Fig. 1, is important for optimizing practical data storage systems based on magneto-optic materials.

Let us emphasize that the effect investigated here is rather minute, as the off-diagonal terms in the permittivity tensor (8) are extremely small. Nevertheless, the GMT is accurate enough to evidence such small changes in the polarization. For example, using the PWE with 540 parameters and 602 boundary discretizations, we find that the maximal residual boundary error is only $0.0033 \%$ for this problem. The values of $S_{3}$ and $S_{4}$, which correspond to field components with an amplitude of approximately $0.1 \%$ of the total field amplitude, are therefore reliable.

As a second numerical example, let us consider a $\mathrm{TiO}_{2}$ ellipsoid. This crystal has extraordinary permittivity $\epsilon_{e}=8.24$ and ordinary permittivity $\epsilon_{o}=6.67$ for the wavelength considered $(633 \mathrm{~nm}){ }^{21}$ The scatterer is illuminated by a $\hat{x}$-polarized plane wave propagating in the $\hat{z}$ direction. The amplitude of the total electric field at time $t=0$ is represented in Fig. 2(a) for the crystal's principal axis parallel to the $\hat{x}$-axis and in Fig. 2(b) for the principal axis parallel to the $\hat{y}$ axis. As the difference between $\epsilon_{e}$ and $\epsilon_{o f}$ is large, the propagation of light strongly depends on the di- rection of the crystal's principal axis. For instance, the principal axis parallel to the incident field polarization [Fig. 2(a)] gives a strong focus at the center of the object. On the other hand, no focus appears when the principal axis is perpendicular to the polarization, and a shadow region is visible behind the scatterer [Fig. 2(b)].

The extension of the GMT to anisotropic media that has been presented here is simple to implement in an existing GMT code. The new expansions converge rapidly and make possible the investigation of subtle phenomena. The present research should find numerous applications in domains in which the interaction of light with anisotropic media is important, for example, in crystal (including semiconductor) optics or practical design of magneto-optic data storage systems.

It is a pleasure to acknowledge fruitful discussions with R. Ballisti, C. Hafner, P. Leuchtmann, and R. Vahldieck. This study was supported by the Swiss National Science Foundation.

\section{References}

1. C. Hafner and L. Bomholt, The $3 D$ Electromagnetic Wave Simulator (Wiley, New York, 1993).

2. Yu. A. Eremin and A. G. Sveshnikov, Electromagnetics 13, 1 (1993).

3. Y. Leviatan, Z. Baharav, and E. Heyman, IEEE Trans. Antennas Propag. 43, 1091 (1995).

4. T. Evers, H. Dahl, and T. Wriedt, Electron. Lett. 32, 1356 (1996).

5. L. Novotny, D. W. Pohl, and B. Hecht, Opt. Lett. 20, 970 (1995).

6. Yu. A. Eremin, N. V. Orlov, and V. I. Rozenberg, Opt. Spectrosc. (USSR) 73, 573 (1992).

7. R. Yew-Siow Tay and N. Kuster, Appl. Computat. Electromagnetics J. 9, 79 (1994).

8. N. B. Piller and O. J. F. Martin, "Extension of the generalized multipole technique to anisotropic medias," Opt. Commun. (to be published).

9. C. Hafner, J. Opt. Soc. Am. A 12, 1057 (1995).

10. M. Kawano, H. Ikuno, and M. Nishimoto, Inst. Electron. Info. Commun. Eng. Trans. Electron. E76-C, 1358 (1996).

11. J. D. Jackson, Classical Electrodynamics, 2nd ed. (Wiley, New York, 1975), Chap. 16.

12. P. A. Bobbert and J. Vlieger, Physica A 137, 209 (1986).

13. J. A. Stratton, Electromagnetic Theory (McGraw-Hill, New York, 1941), Eq. (7.60), p. 410.

14. W. C. Chew, Waves and Fields in Inhomogeneous Media (Institute of Electrical and Electronics Engineers, Piscataway, N.J., 1994), Chap. 1.3.3.

15. E. M. Purcell and C. R. Pennypacker, Astrophys. J. 186, 705 (1973).

16. N. B. Piller, Opt. Lett. 22, 1674 (1997).

17. W. A. McGahan, Z. S. Shan, and J. A. Woollam, Appl. Phys. Commun. 8, 209 (1988).

18. C. F. Bohren and D. R. Huffman, Absorption and Scattering of Light by Small Particles (Pergamon, New York, 1980), p. 63.

19. M. J. Freiser, IEEE Trans. Magn. MG-4, 152 (1965).

20. P. S. Pershan, J. Appl. Phys. 38, 1482 (1967).

21. W. J. Tropf, M. E. Thomas, and T. J. Harris, in Handbook of Optics, M. Bass, ed. (McGraw-Hill, New York, 1995), Vol. 2, Chap. 33. 